\title{
THE MAXIMUM TERM OF AN ENTIRE SERIES WITH GAPS
}

\author{
J. D. BUCKHOLTZ
}

Let $\sum a_{p} z^{p}$ denote the power series for an entire function of order $\rho$ and lower order $\lambda$. S. M. Shah [2] has shown that

$$
\begin{aligned}
& \liminf _{r \rightarrow \infty}[\mu(r)]^{1 / \nu(r)} \leqq e^{1 / \rho}, \\
& \limsup _{r \rightarrow \infty}[\mu(r)]^{1 / v(r)} \geqq e^{1 / \lambda},
\end{aligned}
$$

where $\mu(r)$ denotes the maximum term of $\sum\left|a_{p}\right| r^{p}$ and $\nu(r)$ is the largest integer $p$ for which $\mu(r)=\left|a_{p}\right| r^{p}$.

The object of the present note is to obtain a sharper form of (1) for those entire series which possess Hadamard gaps. For this purpose let the subsequence $\left\{a_{p_{m}}\right\}$ contain all the nonvanishing terms of $\left\{a_{p}\right\}$, and suppose that

$$
\liminf _{m \rightarrow \infty} \frac{p_{m+1}}{p_{m}} \geqq 1+\theta>1
$$

We shall prove the following

ThEOREM. Suppose $\sum a_{p_{m}} z^{p_{m}}$ is an entire series of order $\rho$ and lower order $\lambda$ whose gaps satisfy (2). Then

$$
\begin{aligned}
& \liminf _{r \rightarrow \infty}[\mu(r)]^{1 / v(r)} \leqq \alpha^{1 / \rho}, \\
& \limsup _{r \rightarrow \infty}[\mu(r)]^{1 / \nu(r)} \geqq \beta^{1 / \lambda},
\end{aligned}
$$

where

$$
\alpha=(1+\theta)^{1 / \theta}
$$

and

$$
\beta=(1+\theta)^{(1+\theta) / \theta} .
$$

We call attention to the fact that a series which satisfies (2) need not be of irregular growth; much larger gaps are needed [3] to insure that $\lambda<\rho$.

PROOF. The function $\nu(r)$ is a nondecreasing step function which is continuous from the right and assumes only nonnegative integer

Received by the editors October 14, 1963. 
values. Therefore there is a nondecreasing sequence $\left\{R_{k}\right\}$ which $\nu(r)$ "counts," i.e.,

$$
\nu(r)=\sum_{R_{k} \leq r} 1
$$

For convenience we assume that $R_{1}=1$. No generality is lost since this is equivalent to requiring that

$$
\left|a_{0}\right|=\max _{p \geqq 1}\left|a_{p}\right|
$$

For each $k \geqq 1$, let

$$
t_{k}=\log R_{k}-\frac{\log R_{1}+\cdots+\log R_{k}}{k}
$$

and

$$
u_{k}=\log R_{k+1}-\frac{\log R_{1}+\cdots+\log R_{k}}{k}
$$

In addition to satisfying (2), we assume the sequence

$$
p_{0}, p_{1}, \cdots, p_{m}, \cdots
$$

is such that $p_{0}=0$ and $p_{1}=1$. For notational convenience we shall always denote $p_{m}$ by $n$.

The following relations are easily verified by examining the local minima and maxima of the quantities involved:

$$
\begin{aligned}
\liminf _{m \rightarrow \infty} t_{n} & =\liminf _{r \rightarrow \infty} \frac{\log \mu(r)}{\nu(r)}, \\
\limsup _{m \rightarrow \infty} u_{n} & =\limsup _{r \rightarrow \infty} \frac{\log \mu(r)}{\nu(r)}, \\
\liminf _{m \rightarrow \infty} \frac{\log R_{n}}{\log n} & =\liminf _{r \rightarrow \infty} \frac{\log r}{\log \nu(r)}=\frac{1}{\rho}, \\
\limsup _{m \rightarrow \infty} \frac{\log R_{n+1}}{\log n} & =\limsup _{r \rightarrow \infty} \frac{\log r}{\log \nu(r)}=\frac{1}{\lambda} .
\end{aligned}
$$

We shall also need estimates for the quantities

$$
A(n)=1+\sum_{j=1}^{m-1}\left[\frac{p_{j+1}}{p_{j}}-1\right]
$$

and 


$$
B(n)=1+\sum_{j=1}^{m-1}\left[1-\frac{p_{j}}{p_{j+1}}\right] .
$$

For this purpose let

$$
x_{j}=\frac{p_{j+1}}{p_{j}}-1, \quad j=1,2,3, \cdots .
$$

Then

$$
\log n=\sum_{j=1}^{m-1} \log \left(1+x_{j}\right) .
$$

From (2) and the fact that $(1 / x) \log (1+x)$ is a decreasing function, we obtain

$$
\limsup _{m \rightarrow \infty} \frac{\log n}{A(n)} \leqq \frac{\log (1+\theta)}{\theta} .
$$

A similar argument shows that

$$
\liminf _{m \rightarrow \infty} \frac{\log n}{B(n)} \geqq \frac{(1+\theta) \log (1+\theta)}{\theta} .
$$

Having taken care of the above preliminaries, we turn now to the main body of the proof. Inverting the systems of equations (4a) and (4b) yields (since $R_{1}=1$ )

$$
\log R_{n}=t_{n}+\sum_{k=2}^{n} \frac{t_{k}}{k-1}
$$

and

$$
\log R_{n+1}=u_{n}+\sum_{k=1}^{n-1} \frac{u_{k}}{k+1} .
$$

The values assumed by $\nu(r)$ are all terms of $\left\{p_{m}\right\}$; therefore

$$
\log R_{k}=\log R_{p_{j+1}}, \quad p_{j}<k<p_{j+1},
$$

from which it follows that

$$
t_{k}=\frac{p_{j+1}}{k} t_{p_{j+1}}, \quad p_{j}<k \leqq p_{j+1}
$$

and

$$
u_{k}=\frac{p_{j}}{k} u_{p_{j}}, \quad p_{j} \leqq k<p_{j+1} .
$$


Substituting these expressions in (5a) and (5b), we obtain

$$
\log R_{n}=t_{n}+\sum_{j=1}^{m-1} t_{p_{j+1}}\left[\frac{p_{j+1}}{p_{j}}-1\right]
$$

and

$$
\log R_{n+1}=u_{n}+\sum_{j=1}^{m-1} u_{p_{j}}\left[1-\frac{p_{j}}{p_{j+1}}\right] .
$$

From (6a) and (6b) it follows (cf. [1, p. 52, Theorem 9]) that

$$
\liminf _{m \rightarrow \infty} t_{n} \leqq \liminf _{m \rightarrow \infty} \frac{\log R_{n}}{A(n)}
$$

and

$$
\limsup _{m \rightarrow \infty} u_{n} \geqq \limsup _{m \rightarrow \infty} \frac{\log R_{n+1}}{B(n)} .
$$

From (7a) we have

$$
\begin{aligned}
\liminf _{r \rightarrow \infty} \frac{\log \mu(r)}{\nu(r)} & \leqq \liminf _{m \rightarrow \infty} \frac{\log R_{n}}{A(n)} \\
& \leqq\left[\liminf _{m \rightarrow \infty} \frac{\log R_{n}}{\log n}\right]\left[\limsup _{m \rightarrow \infty} \frac{\log n}{A(n)}\right] \\
& \leqq \frac{\log (1+\theta)}{\rho \theta}
\end{aligned}
$$

Therefore

$$
\liminf _{r \rightarrow \infty}[\mu(r)]^{1 / p(r)} \leqq \alpha^{1 / p}
$$

The remaining portion of the theorem follows similarly from (7b).

We note that $\alpha$ and $\beta$ tend respectively to 1 and $\infty$ as $\theta \rightarrow \infty$. In conjunction with our theorem this remark implies the following

CoRollary. Suppose that $\sum a_{p_{m}} z^{p_{m}}$ is an entire function of positive finite order, and

$$
\lim _{m \rightarrow \infty} \frac{p_{m+1}}{p_{m}}=\infty
$$

Then

$$
\liminf _{r \rightarrow \infty}[\mu(r)]^{1 / \nu(r)}=1
$$


and

$$
\limsup _{r \rightarrow \infty}[\mu(r)]^{1 / \nu(r)}=\infty .
$$

\section{REFERENCES}

1. G. H. Hardy, Divergent series, Clarendon Press, Oxford, 1949.

2. S. M. Shah, The maximum term of an entire series, Math. Student 10 (1942), 80-82.

3. J. M. Whittaker, The lower order of integral functions, J. London Math. Soc. 8 (1933), 20-27.

University of North Carolina

\section{MIXED BOUNDARY-VALUE PROBLEMS IN THE PLANE ${ }^{1}$}

J. A. VOYTUK AND R. C. MAC CAMY

Let $R$ be a region in the plane bounded by a simple analytic curve $C$ composed of $N$ arcs $C_{1} \cdots C_{N}$. Let $a_{m}, b_{m}, f_{m}$ be analytic functions on $C_{m}$. Suppose $q(x, y)$ is non-negative in $R$. The mixed boundaryvalue problems discussed here require the determination of a solution of

$$
\begin{array}{rlrl}
\Delta u-q u & =0 & & \text { in } R, \\
a_{m} u_{n}-b_{m} u=f_{m} & & \text { on } C_{m},
\end{array}
$$

$n$ the exterior normal. The problem is called regular if on each $C_{m}$ either

$$
a_{m}>0, \quad b_{m} \geqq 0
$$

or

$$
a_{m} \equiv 0, \quad b_{m}>0 .
$$

This note presents an existence theorem based on integral equations. The method is an extension of the solution of the Dirichlet problem by simple layers as in [1] and [4]. It is intended also to provide information as to the behavior of $u$ at the ends of the $C_{k}$.

TheOREM 1. Every regular mixed problem has a unique solution.

Received by the editors September 28, 1963.

1 This work was supported by the Air Force Office of Scientific Research and the National Science Foundation. 